\title{
Degassing cascades in a shear-thinning viscoelastic fluid
}

\author{
Valérie Vidal, François Soubiran, Thibaut Divoux and Jean-Christophe Géminard \\ Université de Lyon, Laboratoire de Physique, École Normale Supérieure de Lyon, \\ CNRS, 46 Allée d'Italie, 69364 Lyon cedex 07, France.
}

(Dated: November 8, 2018)

\begin{abstract}
We report the experimental study of the degassing dynamics through a thin layer of shear-thinning viscoelastic fluid (CTAB/NaSal solution), when a constant air flow is imposed at its bottom. Over a large range of parameters, the air is periodically released through series of successive bubbles, hereafter named cascades. Each cascade is followed by a continuous degassing, lasting for several seconds, corresponding to an open channel crossing the fluid layer. The periodicity between two cascades does not depend on the injected flow-rate. Inside one cascade, the properties of the overpressure signal associated with the successive bubbles vary continuously. The pressure threshold above which the fluid starts flowing, fluid deformation and pressure drop due to degassing through the thin fluid layer can be simply described by a Maxwell model. We point out that monitoring the evolution inside the cascades provides a direct access to the characteristic relaxation time associated with the fluid rheology.
\end{abstract}

PACS numbers: 05.45.-a, 47.57.-s, 83.80.Qr, 83.60.Rs

\section{INTRODUCTION}

Non-Newtonian fluids peculiar dynamics has focused a large attention over the last decades. Their ability to flow or break [1, 2], to exhibit viscous or elastic behavior [3], thixotropy effects [4 6] or to sustain loads without flowing [7, 8] opened a wide range of applications, including cosmetics, food industry, environment and biology. Among them, micellar fluids differ from polymers by their internal dynamics: they continuously break and restructurate 9 11], and their internal kinetics determines their length distribution - and, hence, their macroscopic properties [12, 13].

The behavior of bubbles rising through such fluids is complex, due, in particular, to the non-trivial coupling between the bubble and the fluid rheology [14]. This has prevented, up to now, a complete theoretical description of the system, and favored experimental studies. Due to the viscoelastic properties, the bubble shape is generally elongated [3, 14 17], its tail ends by a cusp [14, 15], and both its geometry and velocity oscillate during its rising through the fluid [15, 16, 18]. In shear-thinning fluids, the local perturbation due to a rising bubble - or a falling sphere - creates a negative wake [19] and a corridor of reduced viscosity [20, 21]. As a consequence, successive bubbles may interact one with the other 22 25], if the emission period is shorter than the time for the perturbation created by the bubble to vanish.

An extensive study of the degassing regimes when a constant air flux is imposed at the bottom of a complex, yield-stress fluid column (gel or immersed granular material) has revealed the existence of three different regimes 25 27]. On the one hand, at low flow-rate, bubbles rise independently one from the other. On the other hand, at high flow-rate, an open channel connects the bottom nozzle to the fluid surface; this channel develops instabilities, forming a 'bubble chain' 28]. Finally, at intermediate flow-rate, the system spontaneously oscillates between the two previous regimes, exhibiting a complex intermittent dynamics [25].

In this article, we extend these previous works to the case of a fluid without yield stress, and for a thin fluid layer, i.e., when the fluid height is smaller than the typical size of a bubble. We report the existence of a peculiar degassing regime in a thin layer of micellar fluid (CTAB/NaSal mixture). When a constant air flow is imposed at its bottom, in the bubbling regime, the air is released through periodic series of successive bubbles, hereafter named cascades. When the layer is thin enough, the cascades merely consists in successive opening and closing of the fluid layer above the air injection point. For convenience, these periodic degassing apertures will also be named bubbles in the following. We measure the overpressure at the base of the fluid column, at the injection point. The main characteristics of the cascades (periodicity, maximum overpressure) are analyzed in regards with the fluid rheology. We show that inside a cascade, the bubble properties vary continuously: the mean overpressure drops, whereas the emission time increases.

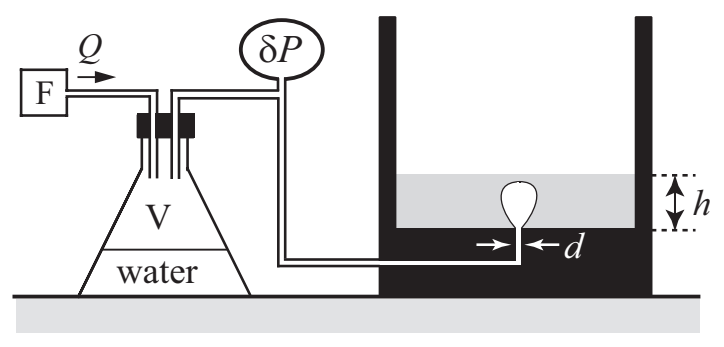

FIG. 1. Experimental setup. A constant air-flow is supplied at the base of a thin layer of micellar fluid by an air-flow controller $\mathbf{F}$ (flow-rate $Q$ ), via a chamber of volume $V . h$ is of the order of a typical bubble diameter (a few millimeters to centimeters). 
The transition between two regimes inside the cascades is directly linked to the fluid rheology and, in particular, provides a direct measurement of the viscoelastic characteristic time associated with the Maxwell fluid.

\section{EXPERIMENTAL SETUP}

The experimental cell consists of a cylinder made of plexiglass (diameter $74 \mathrm{~mm}$, height $270 \mathrm{~mm}$ ), filled with the fluid up to a height $h$ (Fig. 1). Air is injected at constant flow-rate $Q$ (from 0.17 to $1.72 \mathrm{~mL} / \mathrm{s}$ ) through an injection hole (diameter $d=2 \mathrm{~mm}$ ) at the bottom of the fluid column. The air-injection system consists of a massflow controller (Bronkhorst, Mass-Stream series D-5111) connected to a chamber of volume $V$, from which the air flows at the column bottom. The volume of the chamber can be easily tuned by changing the water level into the chamber (see Fig. 11). The water itself makes it possible to inject humid air inside the fluid, thus avoiding any drying of the sample over the experimental time. A differential pressure sensor (223 BD-00010 AB, MKS Instruments) measures the variations of the overpressure $\delta P$ inside the chamber, corresponding to the pressure variations at the bottom of the cell.

The fluid is a semi-dilute micellar system obtained by a mixing at equimolar concentration, inside pure water, sodium salicylate (NaSal, Sigma Aldrich) and hexadecyltrimethylammonium bromide (CTAB, Sigma Aldrich). The mixture of this two chemical components causes the formation of a network of giant entangled micelles, which break down and reform continuously [9]. On a macroscopic point of view, the fluid exhibits shearthinning, viscoelastic properties [29, 30], which can be tuned by varying the fluid concentration $c$ (from 0.03 to $\left.0.5 \mathrm{~mol} . \mathrm{L}^{-1}\right)$. The rheology of these well-controlled mixtures is characterized by rheometer measurements (see Appendix $\mathrm{A}$ and $\mathrm{B}$. The height $h$ of the thin layer ranges from 5 to $35 \mathrm{~mm}$. Unless specified, the results presented here are for a typical height of $5 \mathrm{~mm}$ and a concentration $c=0.1 \mathrm{~mol} . \mathrm{L}^{-1}$.

\section{DEGASSING REGIMES}

When varying the system parameters $(c, V, Q, h)$, we observe three different degassing regimes through the thin layer: a bubbling regime, for which bubbles are emitted one after the other; an open channel regime, for which the system is able to sustain a stable channel connecting the injection nozzle at the base of the fluid column to the fluid free surface; and an intermittent regime, for which the system alternates spontaneously between the bubbling and the open channel regime - this latter pinching off intermittently. Similar degassing regimes have been reported in yield-stress fluids: gels [25] or in immersed granular media [26, 27].

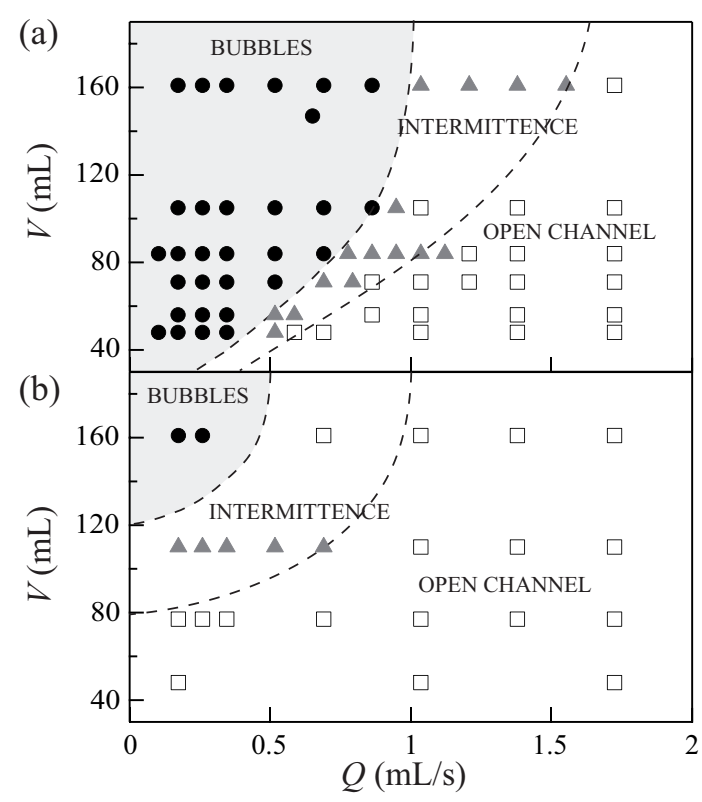

FIG. 2. Phase diagram of the different degassing regimes depending on the parameters $(V, Q)$ for $c=0.1$ mol.L $^{-1}$ (a) and $c=0.5$ mol.L ${ }^{-1}$ (b) $[(\bullet)$ bubbles, $(\boldsymbol{\Delta})$ intermittence, $(\square)$ open channel]. Dashed lines are eyeleads. The gray zone correspond to the space of parameters where bubble cascades are observed $[h=5 \mathrm{~mm}]$.

Typically, when increasing the injected air flow-rate $Q$, all other parameters being constant, the system goes from the bubbling, intermittent and finally open channel regime (Fig. 2). When increasing the fluid concentration, it becomes easier for the system to sustain an open channel, and it is necessary to go to higher volumes $V$ and smaller flow rate $Q$ to observe the bubbling regime (Fig. 2a and b) 31]. On the contrary, increasing the fluid column height makes it more difficult to open a channel, and shifts the regime boundaries toward smaller volumes and higher flow rates. Note that the precise boundary between two different regimes is difficult to determine, due to the finite acquisition time (the system may not have the time to switch from bubbles to an open channel, or vice-versa).

The formation of an open-channel can be qualitatively explained. Indeed, due to the fluid shear-thinning properties (Appendix A), when a bubble rises through the fluid, its wake is characterized by a local viscosity smaller than the surrounding fluid. If the flow rate is large enough, the following bubble will rise through a fluid with a smaller effective viscosity - and thus, will rise faster, and so on, until the system is able to sustain an open channel through the fluid column. Note that this channel does not resemble a cylinder, but rather a bubble chain, similar to previous observations in yield-stress and non yield-stress fluids [25, 28]. 

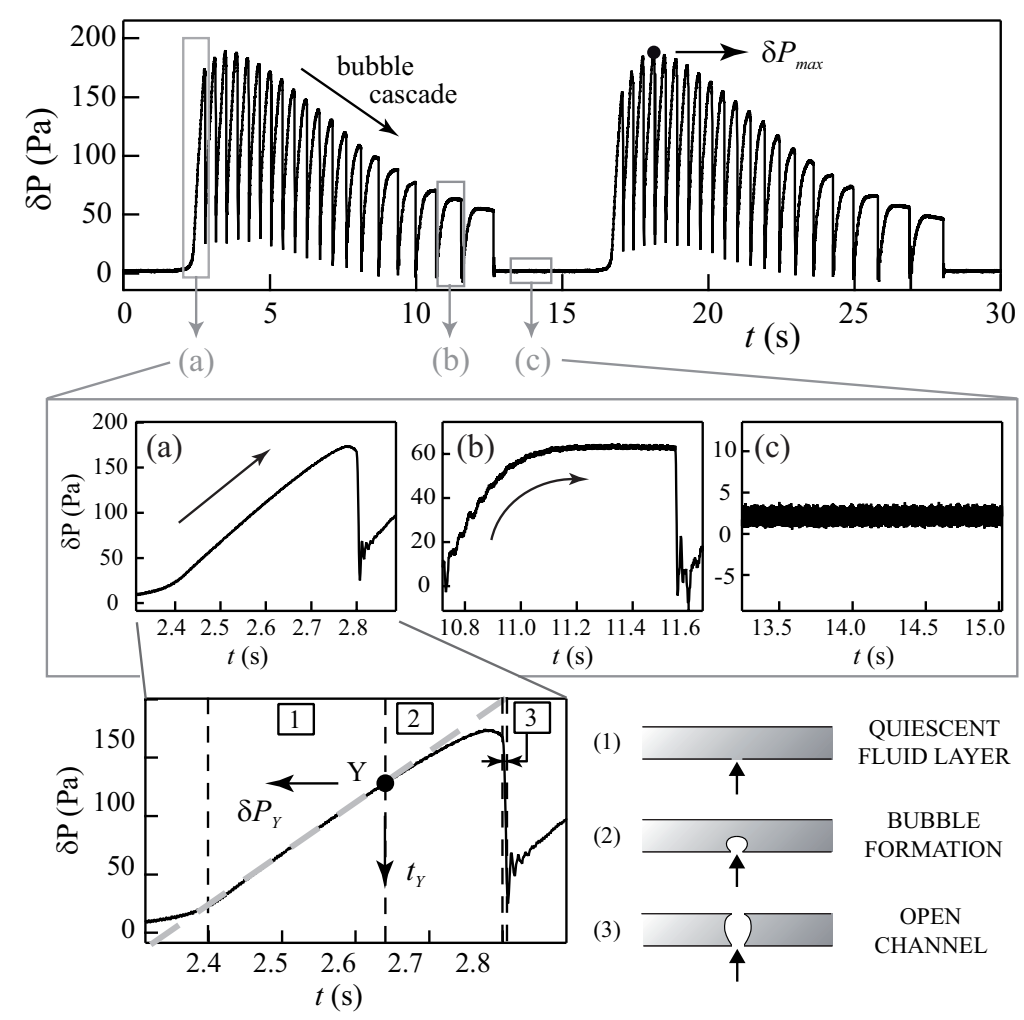

FIG. 3. Top: Pressure signal displaying the gas discharge via bubble cascades. $\delta P_{\max }$ indicates the maximum overpressure reached in the cascades. Middle: (a) The first bubbles in the cascade exhibit a linear pressure increase. The fluid starts flowing only when the bubble is about to be emitted (pressure jump). (b) The last bubbles in the cascade exhibit a curved pressure increase, characteristic of fluid deformation (flowing) and bubble growth. (c) Between two bubble cascades, an open channel connects the air nozzle to the fluid free surface. The overpressure is constant, almost equal to zero (see text). Bottom: Detail of the pressure signal for a bubble formation and emission inside a cascade: (1) linear pressure increase; the dashed gray line corresponds to the linear pressure increase in the fixed volume $V, \delta P=\left(P_{0} / V\right) Q t$, without any ajustable parameters (see text); (2) bubble formation and growth; (3) the bubble pierces the free surface and the gas is released. Note the pressure oscillations subsequent to the bubble emission $\left[c=0.1 \mathrm{~mol} . \mathrm{L}^{-1}, V=147 \mathrm{~mL}, Q=0.65 \mathrm{~mL} / \mathrm{s}\right]$.

\section{BUBBLE CASCADES}

In a wide range of fluid concentration (from $c=0.04$ to $0.14 \mathrm{~mol} / \mathrm{L}$ ), when the fluid layer height $h$ is of the order of the size of a bubble (typically, $h<10 \mathrm{~mm}$ ), we do not observe, in the bubbling regime, successive bubbles but rather periodic series of bubbles, hereafter named bubble cascades. These cascades are clearly observed in the overpressure signal $\delta P$ recorded at the bottom of the fluid column (Fig. 3, top).

The pressure signal corresponding to one cascade exhibits successive rises and drops, each of them corresponding to the pressure increasing in the chamber, followed by a bubble emission (Fig. 3 middle, a and b). After each bubble cascade, the overpressure remains at $\delta P \sim 0$ for a few seconds, before the next bubble cascade (Fig. 3 middle, c). During this time interval, the air escapes continuously through the thin fluid layer. The value of the overpressure is given by the charge loss of the air flow through the hole (roughly a few Pa, Fig. 3 , middle, c). This hole then suddenly closes, and the next cascade starts. The sequence consisting of a bubble cascade followed by a hole opened through the fluid layer repeats periodically in time. The main goal of this work is to describe thoroughly the cascades properties.

The general properties of the cascades are reported in Figure 4. On the one hand, we observe that the time interval between each sequence (cascade + hole) is very stable and, over the range of parameters explored, does not depend neither on the injected flow rate $Q$, nor on the chamber volume $V$. The same observation is reported for the maximum overpressure reached inside each cascade (Fig. 4, inset). On the other hand, the number of bubbles emitted per cascade, $n$, depends linearly on the injected flow rate (Fig. 5a). The associated slope, $d n / d Q$, varies with the chamber volume $V$. For $h=5 \mathrm{~mm}$ and small volumes, we report a linear, decreasing relationship $d n / d Q=-\zeta V$, where $\zeta$ is a constant (Fig. [5b, black dots). For large values of $V(>105 \mathrm{~mL})$ or larger height, however, $d n / d Q$ is independent of the chamber volume. These results can be interpreted as follows.

For small $h$, the layer is of the order of a bubble height, 


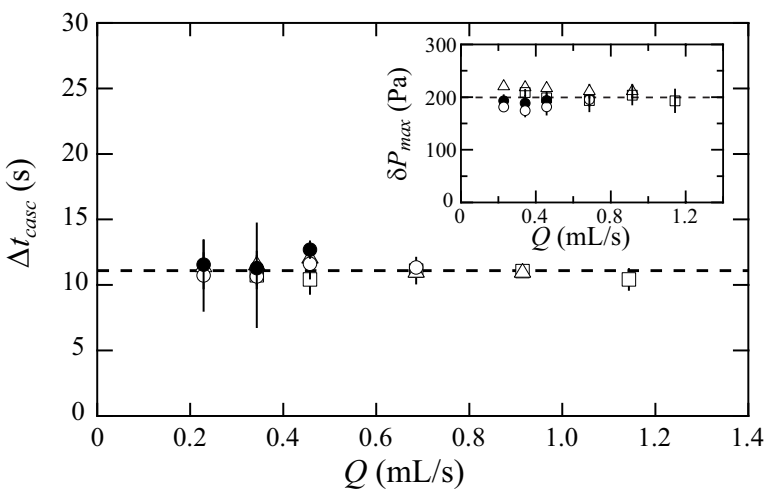

FIG. 4. Time interval $\Delta t_{\text {casc }}$ separating a sequence 'bubble cascade + hole', as a function of the air flow-rate $Q$. Inset : Maximum overpressure $\delta P_{\max }$ reached in the cascades (see Fig. 3). (Symbol, $V[\mathrm{~mL}]):(\bullet, 56),(\circ, 71),(\triangle, 83),(\square, 161)$. Dashed lines indicate the mean value of all the data.

and whenever a bubble is emitted, it pierces the layer. Via the open channel thus formed, the overpressurized air escapes the chamber. For small chamber volume $V$, the chamber reservoir quickly empties, and a dependence on $V$ is clearly seen. When $V$ reaches higher values, it acts as a pressure reservoir and $d n / d Q$ remains roughly constant.

For larger $h$, the air is no longer able to pierce the fluid layer, and bubbles are emitted in the fluid, rise and burst without connecting anymore the injection nozzle to the fluid free surface. In this case, the overpressurized air trapped in the chamber cannot escape directly through the fluid, but via the successive bubbles. The bubble size is fixed by the nozzle size and the fluid rheological properties, and $d n / d Q$ is independent of $V$.

The total volume of gas emitted during a cascade can be written $V_{T} \sim Q \Delta t_{c a s c}$. As the number of bubbles emitted per cascade always depends linearly on $Q$, we can write $n=\alpha Q$, where $\alpha=d n / d Q$ is a constant for a given series of experiment ( $c, h$ and $V$ fixed). We thus find that the average gas volume emitted per bubble, $\left\langle v_{b}\right\rangle=V_{T} / n$, is constant. For $h>5 \mathrm{~mm}$, this constant

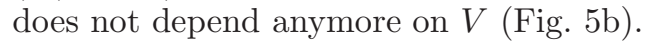

Inside a cascade, however, the bubble properties (maximum overpressure and emission duration) vary continuously (Fig. 3 top and middle). In the next two sections, we investigate these variations, and see which informations they bring on the system.

\section{EVOLUTION INSIDE A CASCADE}

During the release of a single bubble, the overpressure $\delta P$ exhibits three different stages (Fig. 3 bottom). First, we observe a linear pressure increase (Fig. 3, bottom, region 1). The overpressure signal then departs from the linear tendency (Fig. 3. bottom, region 2) until the bubble is emitted (sudden pressure drop, Fig. 3, bottom,
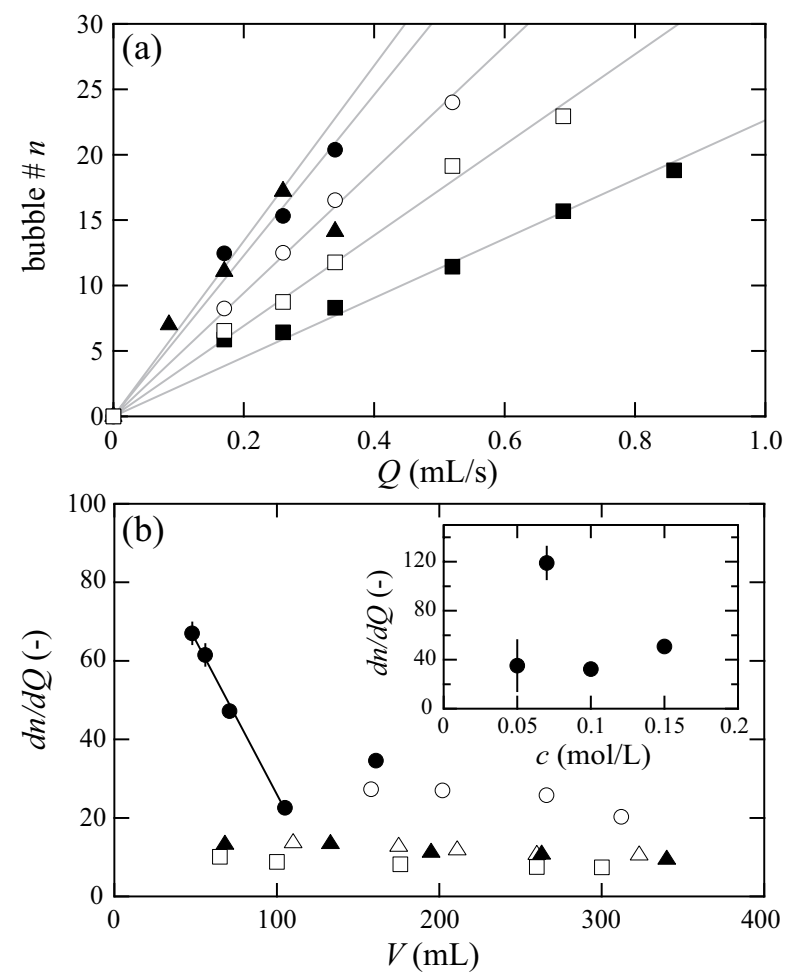

FIG. 5. (a) Number of bubbles per cascade $n$ as a function of the air flow rate $Q$. (Symbol, $V[\mathrm{~mL}])$ : $(\boldsymbol{\Lambda}, 48),(\bullet, 56),(\circ, 71)$,

$(\mathbf{\square}, \mathbf{1 0 5}),(\square, 161)$. The gray lines correspond to the linear interpolation for each series of experiments ( $V$ fixed) $[h=$ $5 \mathrm{~mm}]$. (b) $d n / d Q$ as a function of $V$. The series reported in (a) display a linear, decreasing relationship up to $V=105 \mathrm{~mL}$. For larger fluid layer height, the slope is almost constant. (Symbol, $h[\mathrm{~mm}]):(\bullet, 5) ;(\circ, 8) ;(\triangle, 18) ;(\square, 27) ;(\boldsymbol{\Lambda}, 35)$. Inset: $d n / d Q$ as a function of the fluid concentration $[V=147 \mathrm{~mL}$, $Q=0.65 \mathrm{~mL} / \mathrm{s}, h=5 \mathrm{~mm}$. No apparent relationship is found.

region 3). In this section, we describe each part of the pressure signal, and show that a simple Maxwell model can account for the different observations.

\section{A. Linear pressure increase}

When submitted to a sudden stress (pressure increase), at a short time scale, the $\mathrm{CTAB} / \mathrm{NaSal}$ mixture does not flow, and the system is equivalent to a chamber of volume $V$ continuously filled by a gas flow $Q$. The overpressure is given by

$$
\delta P=\left(\frac{P_{0}}{V}\right) Q t
$$

where $P_{0}=10^{5} \mathrm{~Pa}$ denotes the atmospheric pressure. The experimental slope is consistent with this linear pressure increase (dashed gray line, Fig. 3 bottom, region 1), without any adjustable parameters. In the following section, we estimate the threshold pressure $\delta P_{Y}$ above which 


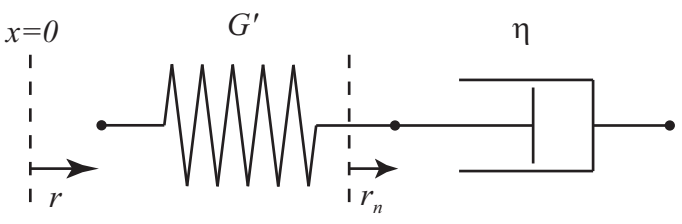

FIG. 6. Maxwell model for the CTAB/NaSal. This simple model consists in an ideal elastic spring (elastic modulus $G^{\prime}$ ) and a dashpot representing the viscous loss (viscosity $\eta$ ). $r$ and $r_{n}$ indicate the displacements generated by the rising bubble and the dashpot shift after the $n^{\text {th }}$ bubble, respectively.

the fluid starts flowing when the pressure at its bottom increases.

\section{B. Threshold pressure $\delta P_{Y}$}

After a certain time $t_{Y}$, associated with an overpressure $\delta P_{Y}$ (point $Y$, Fig. 3 , bottom), the overpressure departs from its linear increase. At this point, the fluid starts flowing, and a bubble is nucleated and grows at the tip of the injection hole (Fig. 3. bottom, region 2). In order to estimate this threshold pressure, we describe the fluid with a Maxwell model, consisting of an ideal elastic spring attached to an ideal dashpot (Fig. 6). This simple model represents the viscoelastic behavior of energy storage and viscous loss, which can be quantified by the elastic $\left(G^{\prime}\right)$ and viscous $\left(G^{\prime \prime}\right)$ modulus, respectively. These modulii can be considered constant as a function of the applied stress in a given frequency range $(\omega \sim 1 \mathrm{~Hz})$ representative of the frequency of bubbles rising through the fluid. The modulii are estimated from the plateau obtained from oscillation measurements (see Appendix B. Fig. (10) to $G^{\prime} \sim 50 \mathrm{~Pa}$ and $G^{\prime \prime} \sim 10 \mathrm{~Pa}$.

The equation describing the opening of a hole of radius $r$ due to a bubble rising in the fluid layer can be written:

$$
\xi \rho r \frac{d^{2} r}{d t^{2}}=-\frac{2 \gamma}{r}-\alpha G^{\prime}\left(r-r_{n}\right)+\delta P-\rho g \Delta h
$$

where $\xi$ is a constant. The first term in the right-hand side represents the closing force due to the fluid surface tension $\gamma$, where $\gamma \simeq 40 \mathrm{mN} / \mathrm{m}$. The second term describes the elastic force which tends to shift the spring back to its initial length, where $\alpha$ is a constant which can be approximated to the inverse of the nozzle radius $2 / d$. $\delta P$ is the pressure inside the chamber (Fig. (1) - and, thus, inside the bubble which starts being generated at the injection nozzle. Finally, the last term quantifies the weight associated with the fluid layer height $\Delta h$ above the newborn bubble. At the limit where the fluid starts flowing, $r \sim d / 2, \Delta h=h$ and the threshold pressure, given by the condition $d^{2} r / d t^{2} \geq 0$, can be written

$$
\delta P_{Y}=\frac{4 \gamma}{d}+\rho g h+\frac{2 G^{\prime}}{d}\left(\frac{d}{2}-r_{n}\right) .
$$

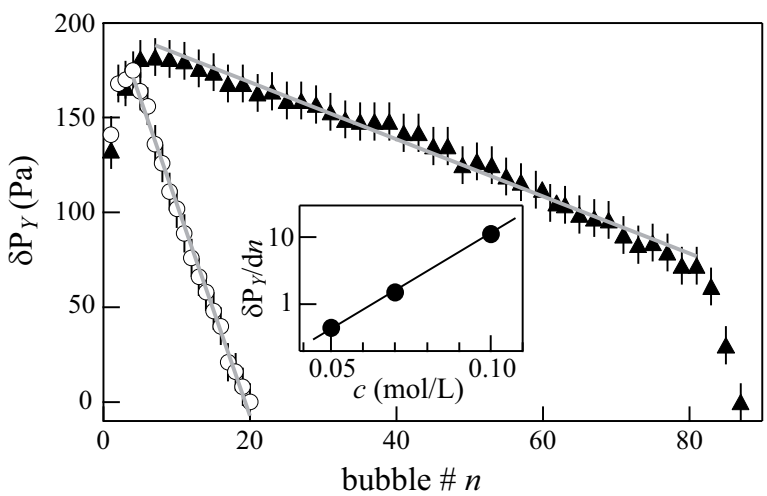

FIG. 7. Evolution of $\delta P_{y}$ as a function of the bubble number in the cascade. In the main part of the cascade, $\delta P_{y}$ is a linear decaying function of the bubble number $n$ (Symbol, $\left.c\left[\mathrm{~mol} . \mathrm{L}^{-1}\right]\right):(\circ, 0.1),(\boldsymbol{\Lambda}, 0.07)[V=147 \mathrm{~mL}, Q=0.65 \mathrm{~mL} / \mathrm{s}]$.

Before the emission of the first bubble in the cascade, $r_{0}=0$ and we can estimate $\delta P_{Y} \sim 180 \mathrm{~Pa}$, which is consistent with the pressure signal measured in the experiments (Fig. 3. top).

As the bubble properties vary continuously inside the cascade, we investigate the evolution of $\delta P_{Y}$ as a function of the bubble number. We find that, in the main part of the cascade, $\delta P_{Y}$ is a linear, decaying function of the bubble number (Fig. 7). The corresponding slope, constant from one cascade to the other, increases with the fluid concentration (Fig. 7, inset). This linear relationship can be explained by a simple heuristic model, based on the key ingredients of the Maxwell model (Fig. 6).

We consider successive bubbles rising through or piercing the thin fluid layer. When the bubble rises during the characteristic time $t_{b}, r \sim d / 2$ (Fig. 6) and we can write the differential equation describing the temporal evolution of $r_{n}$ :

$$
G^{\prime}\left(\frac{d}{2}-r_{n}\right)=\eta \dot{r}_{n}
$$

which gives, right after the bubble rise

$$
r_{n}\left(t_{b}\right)=\left(r_{n-1}-\frac{d}{2}\right) e^{-t_{b} / \tau}+\frac{d}{2}
$$

where $r_{n-1}$ is the initial condition from which $r_{n}$ evolves and $\tau=\eta / G^{\prime}$ the characteristic time associated with the Maxwell fluid.

After the bubble rise, we suppose that the fluid layer closes almost immediately, due in particular to the surface tension. The fluid is hence at rest, and $r_{n}$ relaxes towards 0 during a time $T-t_{b}$, where $T$ is the average time between bubble emission. For sake of convenience, we consider here that $T$ and $t_{b}$ are the same for each bubble. During this stage of relaxation, $r_{n}$ obeys the following equation:

$$
\tau \dot{r}_{n}+r_{n}=0
$$


which gives, after a time $t=T-t_{b}, r_{n}=r_{n}\left(t_{b}\right) e^{-t / \tau}$. We can therefore write the recurrence equation giving the displacement after the $n^{\text {th }}$ bubble:

$$
r_{n}=r_{n-1} e^{-T / \tau}+\frac{d}{2} e^{-T / \tau}\left(e^{-t_{b} / \tau}-1\right) .
$$

By recurrence, and considering that $r_{0}=0$, we thus get the equation describing the displacement $r_{n}$ :

$$
r_{n}=\frac{d}{2}\left(e^{-t_{b} / \tau}-1\right)\left(\frac{e^{-T / \tau}-e^{-n T / \tau}}{1-e^{-T / \tau}}\right) .
$$

For $t_{b}<\tau$ and $T<\tau$ (both the characteristic times of bubble rising through the fluid layer and bubble formation and emission are smaller than the Maxwell time), the slope $d r_{n} / d n$ can be approximated to $(d / 2)\left(t_{b} / \tau\right)$. From Eq. 3. we thus obtain the following expression for the threshold pressure:

$$
\delta P_{Y} \sim\left(\frac{4 \gamma}{d}+\rho g h+G^{\prime}\right)-\frac{1}{2} G^{\prime}\left(\frac{t_{b}}{\tau}\right) n
$$

The average time for a bubble emission (formation and growth, regions 2 and 3 in Fig. 3, bottom) inside a cascade, for $c=0.1$ mol.L $\mathrm{L}^{-1}$, can be estimated to $t_{b} \sim 0.2 \mathrm{~s}$. The characteristic time $\tau=\eta / G^{\prime}$ is obtained from rheological measurements (see Appendix) and can be estimated to $\tau \sim 0.5 \mathrm{~s}$, with $\eta \sim 25 \mathrm{~Pa} . \mathrm{s}$ and $G^{\prime} \sim 50 \mathrm{~Pa}$. We thus estimate a slope $-\left(G^{\prime} / 2\right)\left(t_{b} / \tau\right)$ of about -10 , in agreement with the experimental results (see Fig. 7 for $\left.c=0.1 \mathrm{~mol} . \mathrm{L}^{-1}\right)$.

\section{Fluid deformation}

Above the threshold overpressure $\delta P_{Y}$, the fluid starts flowing and a bubble grows at the tip of the injection hole (Fig. 3, bottom, region 2). The overpressure then departs from its linear increase, as a consequence of the volume increase due to the bubble growth.

By deriving the general equation for an ideal gas, and denoting $v_{b}$ the volume of the bubble hence formed, we get

$$
\frac{d P}{d t}+\left(\frac{P}{V}\right) \frac{d v_{b}}{d t}=\left(\frac{R T}{V}\right) \frac{Q}{V_{m o l}}
$$

Further integration leads to the general expression of the pressure variation in time:

$$
P(t)=\left(\frac{R T}{V}\right)\left(\frac{Q}{V_{\text {mol }}}\right) t-\left(\frac{P}{V}\right) v_{b}
$$

where $R=8.314 \mathrm{~J} \mathrm{~K}^{-1} \mathrm{~mol}^{-1}$ is the ideal gas constant and $V_{m o l}=24.7 \mathrm{~L}$ is the molar volume of air at $25^{\circ} \mathrm{C}$. Here we do not develop further the calculation, but note that the departure from the linear trend is linear with the bubble volume, which provides a rough estimation of this latter, from about $v_{b} \sim 1 \mathrm{~mL}$ to $10 \mathrm{~mL}$ from the beginning to the end of the cascade.

\section{Bubble emission}

Finally, the bubble reaches the free surface - we remind here that the fluid layer is of the order of the bubble size -, the gas is suddenly released and the overpressure quickly drops (Fig. 3, bottom, region 3). In order to get an estimate of the characteristic time over which the pressure drops, we write, on the one hand, Bernoulli's equation to describe the air flowing from the chamber of volume $V$ through the opening of diameter $d$, up to the surface of the fluid layer: $1 / 2 \rho v^{2}=\delta P$, which gives the flow velocity through the opening 32 :

$$
v=\sqrt{\frac{2 \delta P}{\rho}} .
$$

On the other hand, by considering the air as an ideal gas, we get $\delta P / P \sim v_{b} / V$, where $V$ is the initial gas volume, equal to the chamber volume, and $v_{b}$ the volume variation corresponding to the volume of the bubble connected to the injection point. The typical time to empty the chamber can be written $\tau^{*}=\delta V / Q_{v}$, where $Q_{v}=\pi\left(d^{2} / 4\right) v$ is the volumetric flow-rate through the hole. By using Eq. 12, we get the characteristic time for the pressure drop:

$$
\tau^{*}=\left(\frac{V}{P}\right) \frac{1}{\pi d^{2}} \sqrt{\frac{\rho_{a}}{32 \delta P}}
$$

where $\rho_{a}=1.2 \mathrm{~kg} \cdot \mathrm{m}^{-3}$ is the air density, and $P=10^{5} \mathrm{~Pa}$ the atmospheric pressure. $\tau^{*}$ is of the order of a few milliseconds, compatible with the measurements of the pressure drop (region 3 in Fig. 3, bottom).

Note that the drastic pressure decrease due to the hole opening is followed by oscillations observed right after the bubble emission, which correspond to the elastic response of the fluid to the sudden stress imposed by the closing of the bubble walls, after the air release [13].

\section{E. Open channel lifetime}

Here, we develop qualitative arguments to estimate the lifetime of the open channel. We note $r^{*}$ the hole radius for which the channel remains open at the end of a cascade, and $r_{n}^{*}$ the associated value of the inner displacement from the Maxwell model developped above (Fig. 6). When the channel from the injection nozzle to the fluid free surface remains open, $d^{2} r / d t^{2}=0$ and we can write, based on Eq. 2

$$
-\frac{4 \gamma}{d}-G^{\prime}\left(\frac{d / 2-r_{n}^{*}}{d}\right)+\delta P-\rho g h=0
$$

and

$$
-\frac{2 \gamma}{r^{*}}-G^{\prime}\left(\frac{r^{*}-r_{n}^{*}}{d}\right)-\rho g h=0
$$




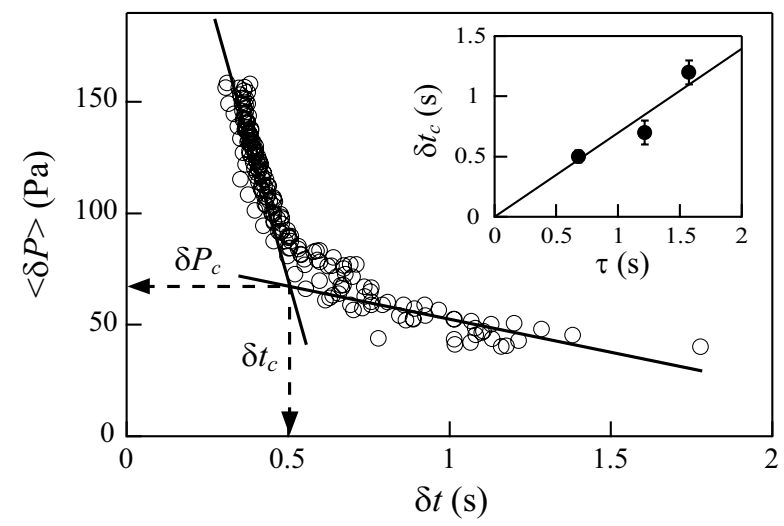

FIG. 8. Mean pressure drop for a bubble vs. time interval for bubble emission. [ $V=147 \mathrm{~mL}, h=5 \mathrm{~mm}, c=0.1 \mathrm{~mol} . \mathrm{L}^{-1}$, $Q=0.65 \mathrm{~mL} / \mathrm{s}]$. Inset: Variations of $\delta t_{c}$ with the fluid characteristic time $\tau$, determined here as $\tau=G^{\prime} / G^{\prime \prime} \omega$ (see text). From left to right: $c=0.1,0.07$ and 0.05 mol.L ${ }^{-1}$.

Eq. 14 provides the value of $r_{n}^{*}$ after the last bubble in the cascade, while Eq. 15 gives the channel radius right after the pressure drop in the system. By substracting Eqs. 14 and 15] and neglecting the capillary forces when the channel if fully open, we find that the channel remains open when the overpressure $\delta P \sim G^{\prime}$. This rough approximation is consistent with the experimental value. Indeed, from Fig. 3] the channel remains open once the overpressure reaches a value close to $50 \mathrm{~Pa}$, of the order of $G^{\prime}$ for an excitation frequency of the order of the frequency of bubbles rising through the fluid $(\omega \sim 1 \mathrm{~Hz}$, see Appendix (B).

After the channel opening, we can write the balance between the main forces at stake, the weight and viscous dissipation, $\eta \dot{r} / d \sim \rho g h / 2$. The characteristic lifetime of the open channel can therefore be roughly estimated to $\tau_{c} \sim 2 \eta / \rho g h$, of the order of a second. Experimentally, $\tau_{c}$ is of a few seconds. This discrepancy can be explained first, by the rough approximation for the radius dynamic equation; then, by the effective fluid viscosity which, for micelles, can be larger during elongational flowing than the viscosity measured under shear [33, 34].

\section{FROM CASCADES TO RHEOLOGY}

The bubble emission characteristics vary continuously inside the cascade: through time, a bubble inside the cascade is emitted with a smaller pressure drop $\delta P$, and a longer emission time $\delta t$ (Fig. 3). Note that for the heuristic model developped in section $\mathrm{VB}$, we considered $\delta t$ roughly constant and equal to $T=\langle\delta t\rangle$. In this section, we investigate the variations of both $\delta t$ and $\delta P$ for the successive bubbles inside one cascade.

Figure 8 displays the mean value of the overpressure $\langle\delta P\rangle$, over a bubble release, as a function of the time interval $\delta t$ over which the same bubble is emitted, for a se- ries of bubble cascades. All data from different cascades, from the same experimental series, have been superimposed. Over different cascades, the data all collapse in the same curve, for a given set of parameters $(c, h, Q$, $V)$. The curves remain unchanged when both the volume $V$ and the flow rate $Q$ vary.

Two different regimes can be distinguished, at short and long time scales, respectively (Fig. 8 solid lines). The limit between both regimes defines a pressure and time threshold, respectively $\delta P_{c}$ and $\delta t_{c}$. A statistical study over different volumes $(56 \leq V \leq 161 \mathrm{~mL})$ and flow rates $(0.2 \leq Q \leq 1.2 \mathrm{~mL} / \mathrm{s})$ gives a constant pressure threshold $\delta P_{c}=71.6 \pm 5.2 \mathrm{~Pa}$. By comparing the characteristic time $\delta t_{c}$ to the Maxwell characteristic time $\tau$ of the fluid (see Appendix), we get the direct relationship

$$
\delta t_{c} \sim \tau .
$$

This relationship holds true for the three different fluid concentrations where bubble cascades are observed (Fig. 8, inset). Measuring the characteristic time linked with the bubble cascades therefore provides a direct signature on the fluid rheology.

\section{CONCLUSION}

Injecting air through a thin layer of micellar fluid displays a wide range of dynamic behavior. We report the existence of a peculiar degassing regime, the bubble cascades regime, for which the air is released via successive bubbles which properties (maximum overpressure and emission duration) vary continuously through time. This regime is observed over a wide range of parameters (air flow-rate $Q$, chamber volume $V$ and fluid height $h)$. The cascades repeat periodically in time, separated by a few seconds during which a channel remains open between the injection nozzle and the fluid free surface, through which the air degass continuously. Measuring the overpressure at the injection point makes it possible to investigate the different stages: pressure increase in the chamber, bubble formation and fluid flow, bubble emission and pressure drop. We find that the cascade periodicity and maximum overpressure depend neither on $Q$ nor $V$. The number of bubbles emitted per cascade depends linearly on the injection flow-rate $Q$. All the different steps of the overpressure evolution can be explained by a simple heuristic model, following the classical Maxwell description of a viscoelastic fluid.

Finally, we underline the interest of such simple experiment. In addition to reporting a new phenomenon (the bubble cascades), we point out that measuring the evolution of the overpressure inside the cascades provides a direct insight into the fluid viscoelastic characteristic time, linked with its rheology. Further work will concentrate on the microscopic behavior of the micelles. In particular, the time over which the rising bubble shears the fluid (typically, $0.2 \mathrm{~s}$ ) suggests a partial alignment of 


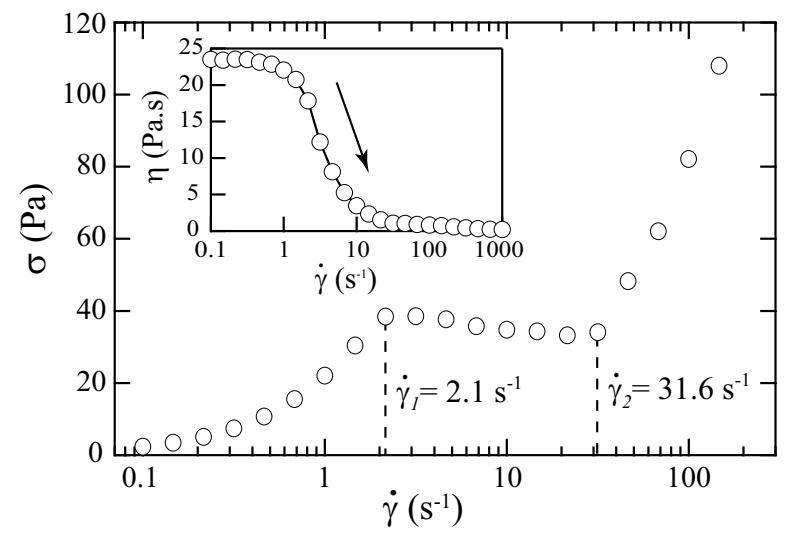

FIG. 9. The flow curve, shear stress $\sigma$ vs shear rate $\dot{\gamma}$ obtained by increasing $\dot{\gamma}\left[c=0.1\right.$ mol.L $\left.\mathrm{L}^{-1}\right]$. Inset: Viscosity as a function of shear rate. The arrow indicates the shearthinning behavior [C-VOR 150 Bohlin rheometer, plate-plate geometry, diameter $60 \mathrm{~mm}$, gap $400 \mu \mathrm{m}$, waiting time $60 \mathrm{~s}$ per point].

the micelles (see Fig. 9, Appendix A). Visualizing the fluid by birefringence will make it possible to determine if the micelles are aligned through time by the shear flow generated by the successive bubbles, and how this alignment is linked with the local rheology. We also propose to investigate the effective fluid viscosity under elongation, which can be much larger than the shear viscosity, and could explain the discrepancy between the estimated and measured time for the open channel lifetime (section $\mathrm{VE})$.

\section{Appendix A: Flow curve}

The rheology of the CTAB/NaSal mixtures is characterized by measurements on two different rheometers: C-VOR 150, Bohlin Instruments and AR1000, TA Instruments. All measurements are performed with a plateplate geometry. Sand paper is glued to the plates in order to prevent any sliding at the walls (typical rugosity of the order of $1 \mu \mathrm{m}$ ). The following results do not aim at a full rheological characterization of the samples used in our experiments. They only provide the general mechanical behavior of the different mixtures, and a support for the interpretation proposed in section VI More detailled informations on the rheology of such systems can be found, for instance, in [9, 10, 12, 13, 29, 30, 35 37.

The semi-dilute solution behaves as a Newtonian fluid at low shear rate $\left(\dot{\gamma}<\dot{\gamma}_{1}=2.1 \mathrm{~s}^{-1}\right)$, with a viscosity plateau $\eta \sim 25 \mathrm{~Pa}$, and exhibits non-Newtonian properties for higher shear rate (Fig. 9). The flow curve is classical for micellar fluids under shear [13, 36], with a plateau in $\sigma$ vs. $\dot{\gamma}$ between $\dot{\gamma_{1}}=2.1 \mathrm{~s}^{-1}$ and $\dot{\gamma_{2}}=31.6 \mathrm{~s}^{-1}$. The first transition in the flow curve $\left(\dot{\gamma}_{1}\right)$ provides an access to the characteristic time associated with the viscoelastic Maxwell model (Fig. [6), $\tau=1 / \dot{\gamma}_{1} \sim 0.5 \mathrm{~s}$.

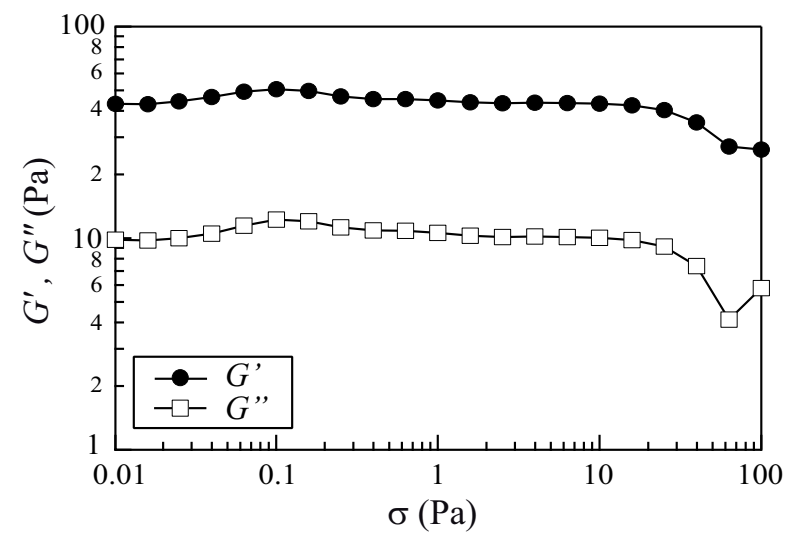

FIG. 10. Elastic $\left(G^{\prime}\right)$ and viscous $\left(G^{\prime \prime}\right)$ modulii as a function of the applied stress for the CTAB/NaSal mixture at $c=$ 0.1 mol.L ${ }^{-1}$ [oscillation test $\omega=1 \mathrm{~Hz}, \mathrm{AR} 1000$ rheometer, plate-plate geometry].

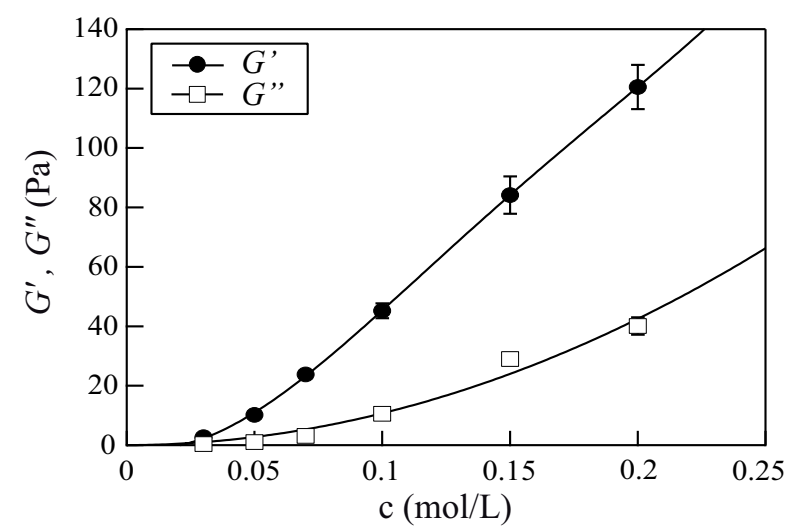

FIG. 11. Average elastic $\left(G^{\prime}\right)$ and viscous $\left(G^{\prime \prime}\right)$ modulii corresponding to the plateau value in Fig. 10] as a function of the fluid concentration. Lines are given as eyeleads.

For $\gamma<\dot{\gamma}_{1}$, the flow is homogeneous and isotropic; for $\dot{\gamma_{1}}<\gamma<\dot{\gamma_{2}}$, shear-bands appear and the flow is strongly inhomogeneous; for $\gamma>\dot{\gamma}_{2}$, finally, the flow is homogeneous and nematic, with the micelles aligned in the shear direction [13]. As the typical time for a bubble to rise up the fluid layer is $t_{b} \sim 0.2 \mathrm{~s}$ (section $\mathrm{VB}$ ), we expect to be in the stress plateau and, therefore, to observe a partial alignment of the micelles (section VII).

\section{Appendix B: Elastic and viscous modulii}

Figure 10 displays the elastic $\left(G^{\prime}\right)$ and viscous $\left(G^{\prime \prime}\right)$ moduli for the fluid $c=0.1$ mol.L ${ }^{-1}$, for an oscillation test ( $\omega=1 \mathrm{~Hz}$, typically the period between two bubbles in our experiments). We observe that over a large range of applied stress, the elastic and viscous modulii are constant. The average plateau values of $G^{\prime}$ and $G^{\prime \prime}$ 
displayed in Fig. 10 provide another estimation of the Maxwell time:

$$
\tau=\frac{G^{\prime}}{G^{\prime \prime} \omega}
$$

We find $\tau \sim 0.5 \mathrm{~s}$ for $c=0.1 \mathrm{~mol} . \mathrm{L}^{-1}$, consistent with the estimation from the previous viscosity measurements (Appendix A).

The average plateau value strongly increases as a func- tion of the fluid concentration (Fig. 111). The values of $\tau$ for different fluid concentrations (Fig. 8 inset, section $(\mathrm{VB})$ have been estimated from the oscillatory measurements and Eq. B1.

Note, finally, that another estimation of the characteristic time $\tau$ can be obtained by

$$
\tau=\frac{\eta}{G^{\prime}}
$$

which, again, gives $\tau \sim 0.5 \mathrm{~s}$, in agreement with the above estimations.
[1] J. Gladden and A. Belmonte, Phys. Rev. Lett. 98, 224501 (2007).

[2] H. Tabuteau, S. Mora, G. Porte, M. Abkarian, and C. Ligoure, Phys. Rev. Lett. 102, 155501 (2009).

[3] R. B. Bird, R. C. Armstrong, and O. Hassager, Dynamics of Polymeric Liquids (Wiley, New York, 1987), Vol. I and II .

[4] J. Mewis, J. Non-Newtonian Fluid Mech. 6, 1 (1979).

[5] H. A. Barnes, J. Non-Newtonian Fluid Mech. 70, 1 (1997).

[6] J. Mewis, and N. J. Wagner, Adv. Coll. Inter. Sci. 147148, 214 (2009) .

[7] P. Coussot, Q. D. Nguyen, H. T. Huynh, and D. Bonn, Phys. Rev. Lett. 88, 175501 (2002).

[8] P. C. F. Möller, J. Mewis, and D. Bonn, Soft Matter 2, 274 (2006).

[9] T. Shikata, H. Hirata, and T. Kotaka, Langmuir 4, 354 (1988).

[10] M. Cates and S. Candau, J. Phys.: Condens. Matter 2, 6869 (1990).

[11] W.M. Gelbart, Micelles, Membranes, Microemulsions and Monolayers (Springer, New York, 1994).

[12] M. Cates, J. Phys. Chem. 94, 371 (1990).

[13] J. F. Berret, Langmuir 13, 2227 (1997).

[14] R. P. Chhabra, Bubbles, Drops and Particles in nonNewtonian Fluids (Chemical industries series \#113, Taylor \& Francis, 2007), 2nd edition.

[15] A. Belmonte, Rheol. Acta 39, 554 (2000).

[16] N. Handzy and A. Belmonte, Phys. Rev. Lett. 92, 124501 (2004).

[17] T. Divoux, V. Vidal, F. Melo, and J.-C. Géminard, Phys. Rev. E 77, 006310 (2008).

[18] A. Jayaraman, and A. Belmonte, Phys. Rev. E 67, 065301(R) (2003).

[19] O. Hassager, Nature 279, 402 (1979).

[20] S. Daugan, L. Talini, B. Herzhaft, and C. Allain, Eur. Phys. J. E 7, 73 (2002).

[21] S. Daugan, L. Talini, B. Herzhaft, and C. Allain, Eur. Phys. J. E 9, 55 (2002).
[22] H. Z. Li, X. Frank, D. Funfschilling, and Y. Mouline, Chem. Eng. Sci. 56, 6419 (2001).

[23] T.-J. Lin and G.-M. Lin, Can. J. Chem. Eng. 81, 476 (2003).

[24] H. Z. Li, X. Frank, D. Funfschilling, and P. Diard, Phys. Lett. A 325, 43 (2004).

[25] T. Divoux, E. Bertin, V. Vidal, and J.-C Géminard, Phys. Rev. E 79, 056204 (2009).

[26] L. Gostiaux, H. Gayvallet, and J.-C Géminard, Gran. Matt. 4, 39 (2002).

[27] G. Varas, V. Vidal, and J.-C. Géminard, Phys. Rev. E 79, 021301 (2009)

[28] I. L. Kliakhandler, Phys. Fluids 14, 3375 (2002).

[29] T. Shikata, H. Hirata, and T. Hotaka, Langmuir 3, 1081 (1987).

[30] T. Inoue, Y. Inoue, and H.Watanabe, Langmuir 21, 1201 (2005).

[31] When the fluid concentration increases, the characteristic time associated with the Maxwell model increases (see Appendix). For the same excitation (bubble rising), the fluid behavior gets closer to an elastic solid response at short time (easier channel opening); at long time, due to the higher viscosity, it is more difficult to close the channel, which is therefore more easily sustained.

[32] G. K. Batchelor, An Introduction to Fluid Dynamics, Cambridge Univ. Press (2000).

[33] R. K. Prud'homme, and G. G. Warr, Langmuir 10, 3419 (1994).

[34] L. M. Walker, and P. Moldenaers, Langmuir 12, 6309 (1996).

[35] T. Shikata, H. Hirata, and T. Kotaka, Langmuir 5, 398 (1989).

[36] S. Lerouge and J.-F. Berret, in Polymer Characterization: Rheology, Laser Interferometry, Electrooptics, edited by K. Dusek and J.-F. Joanny (Springer-Verlag, 2010) pp. 1-171.

[37] J. F. Berret, D. C. Roux, and G. Porte, J. Phys. II France 4, 1261 (1994). 